\title{
The Role of the Endoscopist in the Stroke Unit
}

\author{
Thomas Frieling \\ Department of Gastroenterology, Hepatology, Infectiology, Neurogastroenterology, Hematology, Oncology, and Palliative Medicine, \\ HELIOS-Clinic Krefeld, Krefeld, Germany
}

\section{Keywords}

Acute stroke - Stroke unit - Dysphagia - Endoscopy

Aspiration - Gastrostomy tube feeding

\section{Summary}

Background: Acute cerebral stroke is a common problem that is frequently associated with dysphagia resulting in an increased risk of aspiration pneumonia, complication, and mortality. Methods: A literature review was carried out using the search terms 'stroke unit and endoscopy', 'stroke unit and dysphagia', and 'stroke unit and gastroenterology'. Results: Only few papers focus on the role of endoscopy in stroke units. One major role is the early detection of dysphagia by flexible endoscopic evaluation of swallowing (FEES). FEES appears to have a higher sensitivity and specificity compared to bedside tests and challenges videofluoroscopy. In contrast, other strokeassociated gastrointestinal complications requiring endoscopic diagnostic or therapeutic intervention, such as gastrointestinal bleeding, abdominal pain, or stasis, and the need to provide fluid and food via gastrostomy or jejunostomy are rarely seen in stroke units and become relevant during and after the rehabilitation phase. Conclusion: FEES should involve an interdisciplinary team comprising neurologists, speech and language pathologists and/or therapists, as well as gastroenterologic endoscopists.

(C) 2016 S. Karger GmbH, Freiburg

\section{Introduction}

Stroke is a common problem affecting approximately 40 $50 / 1,000$ people over 55 years of age. Acute stroke produces a wide range of neurological impairments including problems swallowing and eating, lack of postural control, upper-limb dysfunction, visual, cognitive, perceptual and communication impairments, and dyspraxia [1]. The rationale for establishing stroke units is the concept of improving patient outcome by focusing on early rehabilitation as well as intensive monitoring and thrombolytic therapy. From a gastrointestinal point of view, dysphagia, stasis, and malnutrition are the major consequences of acute stroke. These components, alone or in combination, may result in the well-known sequelae of prolonged inpatient care and increased complication and mortality rates $[2,3]$. Therefore, detection and management of stroke-induced gastrointestinal dysfunction must become one of the major focuses in stroke units to improve patient outcome. Here, dysphagia is the predominant problem. In contrast, other stroke-associated gastrointestinal complications requiring endoscopic diagnostic or therapeutic intervention such as gastrointestinal bleeding, abdominal pain, stasis, or malnutrition usually play no significant role in stroke units and are preserved for individual cases; their significance increases during and after the rehabilitation phase. This is illustrated in table 1 indicating the frequency of requests for gastrointestinal endoscopies from the stroke unit at the HELIOS Clinic Krefeld. This analysis shows that gastrointestinal endoscopy was rarely requested from the stroke unit indicating that stroke-induced gastrointestinal complications are infrequent in such units and that nasopharyngeal endoscopy is provided by disciplines other than gastroenterology.

\section{Epidemiology of Stroke-Induced Dysphagia}

Dysphagia occurs frequently and is one of the major disabling factors after acute cerebral stroke. The prevalence of dysphagia in stroke units has been reported to range between 19 [4] and 81\% [5]. Similarly, in a previous study, swallowing abnormalities were detected clinically in 51\% and videofluoroscopically in 64\% [6]. Dysphagia following acute stoke has been associated with an increased risk for pulmonary complications [7]. In a recent review, the reported incidence of dysphagia was lowest using cursory screening techniques (37-45\%), higher using clinical testing (51$55 \%)$, and highest using instrumental testing (64-78\%). There was an increased risk for pneumonia in patients with dysphagia (rela-

\section{KARGER \\ Fax +497614520714

(c) 2016 S. Karger GmbH, Freiburg

$2297-4725 / 16 / 0321-0053 \$ 39.50 / 0$
Prof. Dr. med. Thomas Frieling

Medizinische Klinik II, Innere Medizin mit Gastroenterologie, Hepatologie, Infektiologie, Neurogastroenterologie, Hämatologie, Onkologie und Palliativmedizin

HELIOS-Klinik Krefeld

Lutherplatz 40, 47805 Krefeld, Germany

thomas.frieling@ helios-kliniken.de 
Table 1. Requests from the stroke unit for gastrointestinal endoscopies at HELIOS Clinic Krefeld 2012-2013

\begin{tabular}{llll}
\hline & 2012 & 2013 & 2014 \\
\hline Gastrointestinal endoscopies, n & 11,349 & 11,377 & 11,940 \\
Patients in stroke unit, n & 730 & 694 & 661 \\
Gastrointestinal endoscopies requested by stroke unit, n & 14 & 25 & 46 \\
Esophagogastroduodenoscopy (bleeding, abdominal pain, vomiting, & 13 & 20 & 45 \\
$\quad$ gastrostomy/jejunostomy), n & 1 & 5 & 1 \\
Colonoscopy (bleeding, stasis), n & & & \\
\hline
\end{tabular}

tive risk (RR) 3.17; 95\% confidence interval (CI) 2.07-4.87) and an even greater risk in patients with aspiration (RR 11.56; 95\% CI 3.36-39.77) [8]. Together with dehydration, malnutrition, and other comorbidities, patients are at increased risk of poorer functional outcome and short-term mortality [9]. Despite this impact, available background information on dysphagia, nutrition, and hydration status in acute stroke is limited and conflicting (fig. 1).

\section{Pathophysiology of Stroke-Induced Dysphagia}

Normal swallowing depends on the anatomical and functional integrity of numerous neural structures and extensive pathways in the central and peripheral nervous system (table 2). Lesions in the cerebral cortex, basal ganglia, brain stem, cerebellum, and lower cranial nerves may result in dysphagia. The most common condition associated with dysphagia is stroke. These patients usually have hemiplegia due to lesions in the brain stem or the involvement of one or both hemispheres. On rare occasions, dysphagia may be the sole manifestation of cerebrovascular events such as lacunar infarcts of the periventricular white matter or discrete vascular brain stem lesions. Dysphagia in stroke is usually transient and recovers in almost $90 \%$ of patients within the first 2 weeks. The occurrence of dysphagia does not depend on the site or size of the lesion. However, esophageal motor function shows bilateral cortical representation with consistent interhemispheric asymmetry $[10,11]$. Long persistence of dysphagia following acute stroke appears to be associated with involvement of the right parietal lobe [12]. In addition, a recent review indicated that dysphagia tended to be lower after hemispheric stroke and remained prominent in brain stem stroke patients after rehabilitation [8].

\section{Screening of Dysphagia}

It is evident that clinically the effective identification of patients with dysphagia and malnutrition is a vital first step in appropriate stroke management (fig. 2). There is emerging evidence that early detection of dysphagia in patients with acute stroke reduces not only complications but also length of hospital stay and overall healthcare expenditures [3]. Recent reports indicate that systematic bedside swallowing screening is helpful for the prevention of stroke-associated pneumonia in acute stroke patients in intensive care. For example, early and systematic dysphagia screening using the Gugging Swallowing Screen method reduced the incidence of

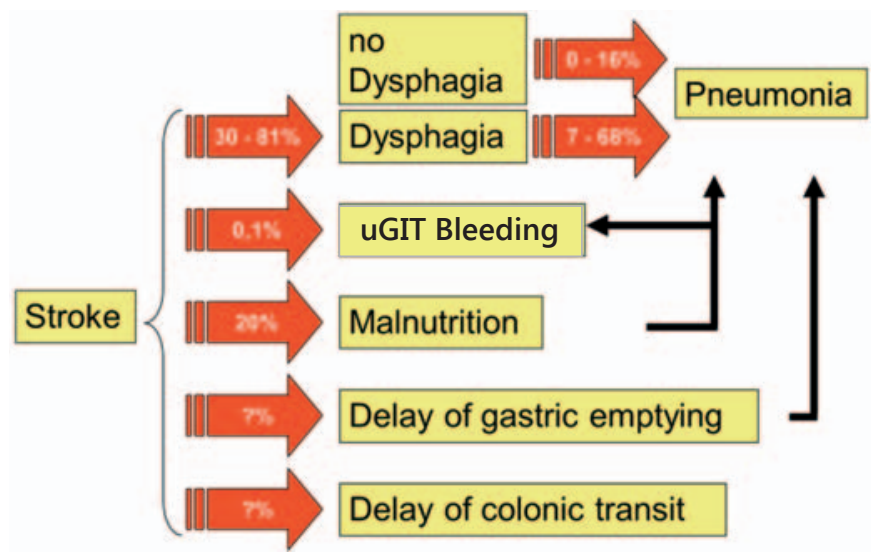

Fig. 1. Consequences of acute cerebral stroke. Occurrence of dysphagia significantly increases the risk of pneumonia $[8,10]$.

Table 2. Physiology of swallowing, pharyngeal phase

Triggering of swallow reflex at the soft palate

Velopharyngeal closure to protect nasal regurgitation

Complete closure of vocal cords

Retroversion of epiglottis

Contraction of constrictor muscles with laryngeal elevation

Laryngeal airway protection
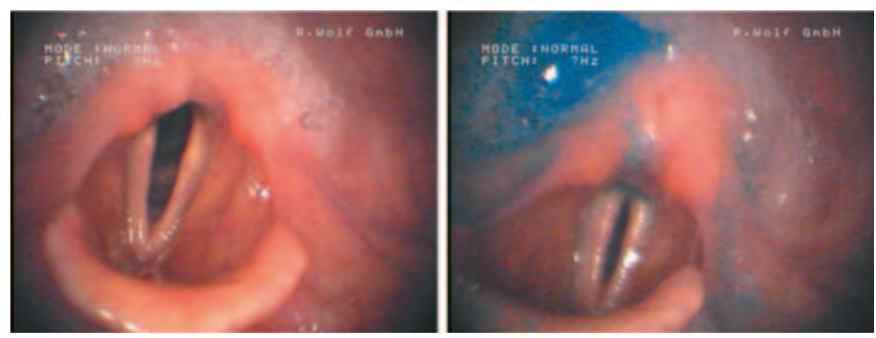

Fig. 2. Oropharyngeal dysphagia following acute stroke. Nasal videoendoscopy shows residual of mucous within both sinus piriformes (left) and predominantly within the right sinus piriformis (right).

X-ray verified pneumonia in the intervention group (7\%) compared to an internal (28\%) and an external (27\%) control group [13], and dysphagia screening using a 3-step swallowing screen decreased the rate of stroke-associated pneumonia [14].

Recognizing the high prevalence of dysphagia following stroke, a measure requiring dysphagia screening prior to oral intake was included in several consensus recommendations. Importantly, this measure required that the screening tool be an 'evidence-based 
Table 3. Results of nasal videoendoscopy, pathophysiology, and therapeutic consequences [22]

\begin{tabular}{|c|c|c|}
\hline Nasal videoendoscopy & Pathophysiology & Therapeutic consequences \\
\hline Premature bolus transit & $\begin{array}{l}\text { disturbed bolus control } \\
\text { delayed reflex triggering }\end{array}$ & $\begin{array}{l}\text { training of autonomic motoric } \\
\text { thermal reflex stimulation }\end{array}$ \\
\hline Residual posterior nasal entrance & incomplete velopharyngeal completion & thermal velum stimulation \\
\hline Residual pharyngeal wall & $\begin{array}{l}\text { insufficient pharyngeal contraction } \\
\text { insufficient propulsive force of tongue } \\
\text { reduced larynx elevation }\end{array}$ & $\begin{array}{l}\text { training of tongue motoric } \\
\text { Masako maneuver }\end{array}$ \\
\hline Residual valleculae & $\begin{array}{l}\text { reduced retraction of basal tongue } \\
\text { reduced larynx elevation }\end{array}$ & training of tongue retraction \\
\hline Residual recessus piriformis & $\begin{array}{l}\text { reduced larynx elevation } \\
\text { reduced opening of upper esophageal sphincter }\end{array}$ & $\begin{array}{l}\text { training of arbitrary larynx elevation } \\
\text { larynx elevation through voice training } \\
\text { Mendelsohn maneuver }\end{array}$ \\
\hline Residual posterior commissure & reduced opening of upper esophageal sphincter & training of larynx elevation \\
\hline Penetration & $\begin{array}{l}\text { reduced larynx elevation } \\
\text { reduced dorsal movement of epiglottis } \\
\text { paresthesia }\end{array}$ & $\begin{array}{l}\text { Mendelsohn maneuver } \\
\text { training of larynx elevation } \\
\text { change of posture } \\
\text { change of bolus consistency }\end{array}$ \\
\hline Aspiration & $\begin{array}{l}\text { reduced closure of laryngeal entrance } \\
\text { insufficient glottis closure } \\
\text { paresthesia } \\
\text { combination of different pathophysiologies }\end{array}$ & $\begin{array}{l}\text { training of vocal cord - adduction } \\
\text { training of supraglottic swallowing }\end{array}$ \\
\hline
\end{tabular}

bedside testing protocol'. Protocols including a checklist and water swallow test resulted in the best patient outcomes $[15,16]$. For example, the Barnes-Jewish Hospital-Stroke Dysphagia Screen (BJHSDS) was previously developed as a simple bedside screen performed by nurses for the sensitive detection of dysphagia, and was previously validated against the speech pathologist's clinical assessment for dysphagia. In a previous study in 225 stroke patients, BJH-SDS had a sensitivity and specificity to detect dysphagia of 94 and $66 \%$, respectively [17]. Sensitivity and specificity of the screen to detect aspiration were 95 and $50 \%$, respectively. No increase in pneumonia was identified during implementation of the screen.

\section{The Role of Endoscopy in Stroke Units}

Several prospective studies have indicated that systematic screening of dysphagia leads to a significant reduction in pneumonia after acute stroke [15]. Therefore, systematic dysphagia screening by protocol has been incorporated in German consensus quality indicators of the German Stroke Registry [18]. However, the significance of clinical dysphagia screening may be compromised by its low sensitivity, specificity, and interobserver reliability. Because of these limitations, flexible endoscopic evaluation of swallowing (FEES) is gaining importance in Germany, even challenging the role of videofluoroscopy as gold standard for the study of swallowing disorders. Indeed, a previous survey indicates that FEES is applied in more than $50 \%$ of the certified stroke units in Germany
[19]. In addition, FEES was recommended for the detection of dysphagia after acute stroke by a German expert group [20]. Flexible nasopharyngeal endoscopy has established itself as the method of choice in the diagnosis of dysphagia of neurological origin. It allows direct visualization of aspiration, is particularly powerful in the analysis of the swallowing process, and usefully informs both the planning and the evaluation of therapy (table 3) [21]. The procedure is generally performed using flexible videoendoscopes that are nasally introduced into the pharynx. Recent studies indicate that FEES is safe and well tolerated by the patients [22]. No significant cardiorespiratory deterioration or severe complications such as vasovagal reaction, laryngospasm, or epistaxis requiring intervention have been reported even within the first $24 \mathrm{~h}$ of medical thrombolysis. Because of this, FEES is less risky compared to placement of nasogastric tubes or nasotracheal suction. A 3-step process is recommended to acquire the relevant knowledge and skills for carrying out FEES [23]. After systematic training (first step), swallowing endoscopy should first be carried out under close supervision (second step), which is then followed by independent practice coupled with indirect supervision (third step). However, neither an educational curriculum for FEES taking into account the specific needs of a stroke unit nor recommendations or guidelines about the qualified application of FEES are currently available. In principle, FEES should adopt a team approach involving both neurologists and speech and language pathologists or alternatively speech therapists. The allocation of responsibilities between these 2 professions should be kept flexible and adjusted to the individual level 
Fig. 3. Algorithm of dysphagia screening in stroke units adopted and modified from [24-26]. KSA = Evaluation of medical history for swallowing; KSU = clinical swallowing assessment.

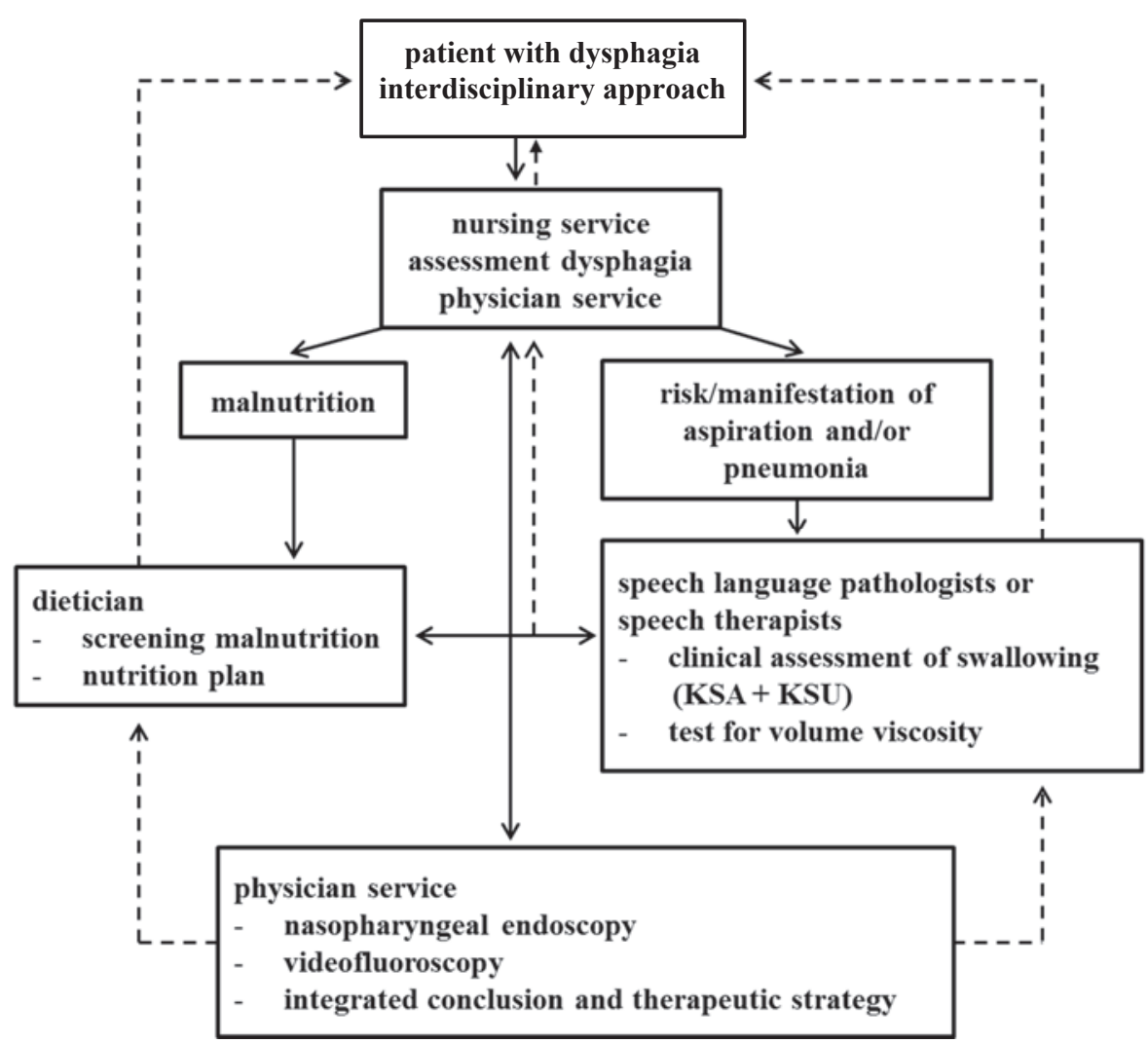

of education. In addition, it seems appropriate to include a gastroenterologist experienced in endoscopy if available. To enhance interprofessional communication and to allow for a smooth and efficient workflow, endoscopic grading of stroke-related dysphagia should adopt a standardized score that also includes protective and rehabilitative measures as well as nutritional recommendations. Nowadays, the systematic evaluation of neurogenic oropharyngeal dysphagia (NOD) in stroke units is based on a 3-step procedure [24-26]. Step 1 is the systematic and standardized evaluation of the patient's history regarding swallowing disorders. Step 2 comprises the clinical assessment of swallowing by consecutive swallow attempts induced by $2 \times 5 \mathrm{ml}$ (teaspoon), $2 \times 10 \mathrm{ml}$, and $2 \times 20 \mathrm{ml}$ of water followed by continuous swallowing of $90 \mathrm{ml}$ (glass) water (step 3).

The role of the endoscopist in the maintenance of the hydration and nutrition needs of patients in stroke units is limited because fluid and food delivery can be achieved safely in most patients with dysphagia following acute stroke. In addition, various studies failed to identify any significant relationship between dysphagia and nutritional status in patients with acute ischemic stroke [27] (table 1). However, direct delivery of food into the stomach or jejunum may be indicated in some cases. The use of gastrostomy tubes is preferred to nasoesophageal intubation because nasogastric tube feeding is usually poorly tolerated and may make the patient irritable or even agitated. Because of this, the volume of food delivered in this way is usually not adequate [10]. Feeding via a gastrostomy tube should be considered when dysphagia is likely to be progressive or to persist for long periods. Intrajejunal delivery of food via a gastrostomy and jejunal feeding tube or via a jejunostomy should be reserved for patients with gastroesophageal reflux or recurrent aspiration on gastrostomy feeding. Interprofessional communication with the gastroenterologist and endoscopist is mandatory here to provide safe and adequate patient care. This is illustrated in figure 3 showing a feasible clinical algorithm for dysphagia screening in stroke units.

In conclusion, the role of the endoscopist in the stroke unit is to be an integral part of an interdisciplinary team also including neurologists and speech and language pathologists or therapists. The major focus of endoscopists in stroke units is to detect and manage stroke-induced dysphagia whereas other requests such as clarification of bleeding, abdominal pain, and stasis, or creation of gastrostomies and jejunostomies are rare.

\section{Disclosure Statement}

The author declared no conflict of interest. 


\section{References}

1 Axelsson K, Norberg A, Asplund K: Eating after a stroke - towards an integrated view. Int J Nursing Stud 1984;21:93-99.

2 Davalos A, Ricart W, Gonzalez-Huix F, et al: Effect of malnutrition after acute stroke on clinical outcome. Stroke 1996;27:1028-1032.

3 Perry L, Love CP: Screening for dysphagia and aspiration in acute stroke: a systematic review. Dysphagia 2001;16:7-18.

4 Barer DH: The natural history and functional consequences of dysphagia after hemispheric stroke. J Neurol Neurosurg Psychiatry 1989;52:236-241.

5 Meng NH, Wang TG, Lien IN, et al: Dysphagia in patients with brainstem stroke: incidence and outcome. Am J Phys Med Rehabil 2000;79:170-175.

6 Mann G, Hankey GJ, Cameron D, et al: Swallowing function after stroke. Prognosis and prognostic factors at 6 months. Stroke 1999;30:744-748.

7 Marik PE, Kaplan D: Aspiration pneumonia and dysphagia in the elderly. Chest 2003;124:328-336.

8 Martino R, Foley N, Bhogal S, et al: Dysphagia after stroke. Incidence, diagnosis, and pulmonary complications. Stroke 2005;36:2756-2763.

9 Sharma JC, Fletcher S, Vassallo M, Ross I: What influences outcome of stroke - pyrexia or dysphagia? Int J Clin Pract 2001;55:17-20.

10 Schaller BJ, Graf R, Jacobs AH: Pathophysiological changes of the gastrointestinal tract in ischemic stroke. Am J Gastroenterol 2006;101:1655-1665.
Aziz Q, Rothwell JC, Hamdy S, et al: The topographic representation of esophageal motor function on the human cerebral cortex. Gastroenterology 1996;111:855862.

12 Bakheit AMO: Management of neurogenic dysphagia. Postgrad Med J 2001;77:694-699.

13 Sørensen RT, Rasmussen RS, Overgaard K, et al: Dysphagia screening and intensified oral hygiene reduce pneumonia after stroke. J Neurosci Nurs 2013;45:139146.

14 Yeh SJ, Huang KY, Wang TG, et al: Dysphagia screening decreases pneumonia in acute stroke patients admitted to the stroke intensive care unit. J Neurol Sci 2011;306:38-41.

15 Hinchey JA, Shephard T, Furie K, et al; Stroke Practice Improvement Network Investigators: Formal dysphagia screening protocols prevent pneumonia. Stroke 2005; 36:1972-1976

16 Suiter DM, Leder SB: Clinical utility of the 3-ounce water swallow test. Dysphagia 2008;23:244-250.

17 Edmiaston J, Connor LT, Steger-May K, Ford AL: A simple bedside stroke dysphagia screen, validated against video-fluoroscopy, detects dysphagia and aspiration with high sensitivity. J Stroke Cerebrovasc Dis 2014; 23:712-716.

18 Heuschmann PU, Biegler MK, Busse O, et al: Development and implementation of evidence-based indications for measuring quality of acute stroke care: the Quality Indicator Board of the German Stroke Registers Study Group (ADSR). Stroke 2006;37:2573-2578.
19 Suntrup S, Meisel A, Dziewas R, et al: Dysphagiediagnostik und -therapie des akuten Schlaganfalls - eine bundesweite Erhebung auf zertifizierten Stroke-Units. Nervenarzt 2012;83:1619-1624.

20 Prosiegel M, Riecker A, Weinert M, et al: Dysphagiemanagement in der akuten Schlaganfallphase. Nervenarzt 2012;83:1590-1599.

21 Kaiser J, Kley J, Uekötter C, et al: Neurogenic dysphagias - the role of videoendoscopy in diagnosis. Dtsch Arztebl 2005;102:A3512-A3516.

22 Dziewas R, Busse O, Glahn J, et al: FEES in the stroke unit. Recommendations for implementation in the clinical routine. Nervenarzt 2013;84:705-708.

23 ASHA: Knowledge and Skills for Speech-Language Pathologists Performing Endoscopic Assessment of Swallowing Functions. American Speech-Language-Hearing Association, 2002.

24 Ickenstein GW: Standardisierung des Untersuchungsablaufs bei Neurogener Oropharyngealer Dysphagie (NOD). Neuro Rehabil 2009;15:290-300.

25 Ickenstein GW: Dysphagie-Management. Modifizierte Version 2012. Handlungsempfehlungen zur Diagnostik und Therapie der neurogenen Dysphagie. HELIOS Kliniken.

26 Ickenstein GW, Hofmayer A, Lindner-Pfleghar B, et al NOD-Stufenkonzept. Neuro Rehabil 2009;15:290-300.

27 Crary MA, Humphrey JL, Carnaby-Mann G, et al Dysphagia, nutrition, and hydration in ischemic stroke patients at admission and discharge from acute care. Dysphagia 2013;28:69-76. 